\title{
TANGENT CONES AND LIPSCHITZ STRATIFICATIONS
}

\author{
TADEUSZ MOSTOWSKI \\ Institute of Mathematics, Warsaw L'niversity, \\ Warsaw, Poland
}

\section{Introduction and statement of the results}

This paper is closely related to [1].

In [3] B. Teissier gave an algebraic characterisation of Whitney's conditions. It would be interesting to have also an algebraic characterisation of stratifications satisfying the estimates of Proposition 1.1 in [1].

We shall do here the first step in this direction, i.e. for a given (germ at 0 of an) analytic set $X \subset C^{n}$ we shall give an algebraic description of all analytic sets $Y$ such that, for some constant $C$,

$$
\left|P_{q_{1}}-P_{q_{2}}\right| \leqslant C\left|q_{1}-q_{2}\right| / \operatorname{dist}\left(\left\{q_{1}, q_{2}\right\}, Y\right)
$$

for all $q_{1}, q_{2} \in X_{\text {reg }}$, where $P_{q}: T_{q} C^{n} \rightarrow T_{q} X$ is the orthogonal projection. It relates the inequality (*) to singular parts of tangent cones to $X$ at points of $X_{\text {sing }}$.

To get an idea of how our characterisation looks like, consider for a moment a hypersurface $X$ given by one equation $F=0, F$ without multiple factors. Let $p \in X_{\text {sing }}$; we have the notion of the tangent cone $C_{p}(X) \subset T_{p} C^{n}$ to $X$ at $p([4])$. It is given by $G_{p}(\xi)=0$, where $G_{p}$ is the homogeneous part of $F(p+\xi)$ and $\xi=\left(\xi_{1}, \ldots, \xi_{n}\right)$. Assume that $G_{p}$ has no multiple factors for all $p$. Let $C_{p}^{\prime}(X)$ be the singular part of $C_{p}(X)$. Then, as we shall prove, a necessary condition for $Y$ to satisfy $(*)$ is

$$
C_{p}^{\prime}(X) \subset C_{p}(Y) \text { for all } p \in X_{\text {sing }},
$$

and this is the only condition for tangent cones to $Y$.

To treat the general case we need a definition, closely related to Zariski's equisingularity.

Consider the space $C^{n}$ with a distinguished hyperplane $H$, given by $\mid x_{1}$ $=0\}$. We shall say that a linear projection $\pi: C^{n} \rightarrow C^{d}$ (where $C^{d}$ is given by $x_{d+1}=0, \ldots, x_{n}=0$ ) is parallel to $H$ if its kernel is spanned by vectors in $H$. 
Let $X \subset C^{n}$ be a hypersurface, given by a reduced equation $F=0$, such that $\operatorname{dim} X \cap H<n-1$. A point $p \in X \cap H$ will be called a $Z$-point of $X$ if, for a generic projection $\pi: C^{n} \rightarrow C^{n-1}$ parallel to $H$, the discriminant of $F$ with respect to $\pi$ is $\neq 0$ for all $x_{1} \neq 0$, in a neighbourhood of $\pi(p)$. (The kernel of any projection. $\pi$, parallel to $H$, contains a unique vector of the form $a$ $=\left(0, a_{2}, \ldots, a_{n-1}, 1\right)$; the space of all such projections can be thus identified with $\boldsymbol{C}^{n-2}$ and genericity means "outside of an algebraic set". If a projection $\pi$ induces a finite map $X \rightarrow C^{n-1}$ and we choose the $x_{n}$-axis so that $\pi\left(x_{1}, \ldots, x_{n}\right)=\left(x_{1}, \ldots, x_{n-1}\right)$, then $F$ is equivalent, in a neighbourhood of $p$, to a distinguished polynomial with respect to $x_{n}$; the discriminant of this polynomial we call the discriminant of $F$ with respect to $\pi$ ).

A point in $X \cap H$ which is not a $Z$-point will be called an $N Z$-point; thus we defined two subsets of $X \cap H: Z(X)$ and $N Z(X)$.

Let $p \in Z(X)$; we choose the $x_{n}$-axis to be the kernel of the generic projection $\pi$. Then $X$ can be described in a neighbourhood of $p$ in terms of Puiseux series:

$$
x_{n}=p_{n}+\varphi_{a}\left(x_{1}^{1 / r}, x_{2}, \ldots, x_{n-1}\right), \quad \alpha=1, \ldots, k,
$$

where $p_{n}$ is the $x_{n}$-th coordinate of $p$ and the analytic functions $\varphi_{a}\left(t, x_{2}, \ldots, x_{n-1}\right)$ satisfy, for every $r$-th root of unity $\varepsilon$,

$$
\begin{aligned}
\varphi_{a}\left(t, x_{2}, \ldots, x_{n-1}\right)-\varphi_{B}\left(\varepsilon t, x_{2}, \ldots, x_{n-1}\right) \\
\text { is either identically } 0 \text { or } \neq 0 \text { for all } x_{1} \neq 0 .
\end{aligned}
$$

Now suppose that $X \subset C^{n}$ is of pure dimension $d$ and $\operatorname{dim} X \cap H<d$. A point $p \in X \cap H$ will be called a $Z$-point of $X$ if for a generic projection $\pi$ : $C^{n}$ $\rightarrow C^{+1}$, parallel to $H$, the point $\pi(p)$ is a $Z$-point of the hypersurface $\pi(X)$; the distinguished hyperplane of $C^{d+1}$ is of course $\left\{x_{1}=0\right\}$.

It is easy to prove that $p$ is a $Z$-point of $X$ if and only if for generic axes $x_{d+1}, \ldots, x_{n}$ in $H$ the coordinates of points of $X$ in a neighbourhood of $p$ satisfy equations of the form

$$
x_{i}=\psi_{i, \alpha}\left(x_{1}^{1 / r}, x_{2}, \ldots, x_{d}\right), \quad \alpha=1, \ldots, \alpha_{i}, i=d+1, \ldots, n,
$$

where, for every $i$, the analytic functions $\varphi_{a}\left(t, x_{2}, \ldots, x_{d}\right)=\psi_{i, a}\left(t, x_{2}, \ldots, x_{d}\right)$ satisfy (2).

Now we return to our problem. Let $X \subset C^{n}$ be of pure dimension $d$ and $p \in X$. Let $M \rightarrow C^{n}$ be the $\sigma$-process centered at $p$ and $M_{0}$ its exceptional fiber. We cover $M$ by open sets $M_{1}, \ldots, M_{n}$ such that $\left(M_{i}, M_{0} \cap M_{i}\right) \approx\left(C^{n},\left\{x_{1}=0\right\}\right)$. Let $\tilde{X}_{p}, \tilde{Y}_{p}$ be the strict transforms of $X$ and $Y$; put

$$
N Z\left(\tilde{X}_{p}\right)=\bigcup_{i} N Z\left(\tilde{X}_{p} \cap M_{i}\right) .
$$

Then a necessary condition for $Y$ to satisfy (*) is

$$
N Z\left(\tilde{X}_{p}\right) \subset \tilde{Y}_{p} \cap M_{0} \quad \text { for all } p \in X_{\text {sing }} \text {. }
$$


This is a condition for the tangent cones $C_{p}(Y)$, for $\tilde{Y}_{p} \cap M_{0}$ can be identified with $C_{p}(Y)$ in the following way. $M_{0} \approx C P^{n-1}$, so $\tilde{Y}_{p} \cap M_{0}$ is a projective variety given, say, by homogenous equations $G_{i}(\xi)=0$. These equations define also a subset of $C^{n}:\left\{p+\xi: G_{i}(\xi)=0\right\}$; this set is $C_{p}(Y)$.

It is easy to check that (4) generalises (1); under the assumptions of (1) $N Z\left(\tilde{X}_{p}\right)$ is identified with $C_{p}(X)$ as $\tilde{Y}_{p} \cap M_{0}$ is identified with $C_{p}(Y)$.

Now $C_{p}(Y)$ can be considered as the "first non-trivial jet" of $Y$ at $p$; this suggests that (4) should be considered as the first of a sequence of conditions for $Y$, of the same nature.

We need some notation. $C^{n}$ with subscripts $C_{0}^{n}, C_{1}^{n}, \ldots$ will all be copies of $C^{n}$ with the distinguished hyperplanes $H_{0}, H_{1}, \ldots$, given by $x_{1}=0$.

For every $i, 1 \leqslant i \leqslant n$, and $p \in C^{n}$ we put

$$
\sigma_{p}^{i}: C_{0}^{n} \rightarrow C^{n}, \quad \sigma_{p}^{i}\left(x_{1}, \ldots, x_{n}\right)=p+\left(x_{1} x_{i}, \ldots, x_{i-1} x_{i}, x_{i}, x_{i} x_{i+1}, \ldots, x_{i} x_{n}\right)
$$

( $\sigma$-process). For every $a \in N$ let

$$
\psi^{a}: C_{1}^{n} \rightarrow C_{0}^{n}, \quad \psi^{a}\left(x_{1}, \ldots, x_{n}\right)=\left(x_{1}^{a}, x_{2}, \ldots, x_{n}\right) .
$$

For $p \in C_{j}^{n}(j \geqslant 1)$ let $\sigma_{p}$ be again the $\sigma$-process

$$
C_{j+1}^{n} \ni\left(x_{1}, \ldots, x_{n}\right) \mapsto p+\left(x_{1}, x_{1} x_{2}, \ldots, x_{1} x_{n}\right) \in C_{j}^{n}
$$

For an equidimensional $X \subset C^{n}$ we put

$$
\begin{aligned}
X_{0}^{i}(p) & =\text { strict transform of } X \text { under } \sigma_{p}^{i}, \\
X_{0}^{\wedge i}(p) & =N Z\left(X_{0}^{i}(p)\right) \subset H_{0}, \\
X_{0}^{i} & =\left\{\left(p, p_{0}\right): p \in X_{\text {sing }}, p_{0} \in X_{0}^{i}(p)\right\} \subset X_{\text {sing }} \times C_{0}^{n}, \\
X_{0}^{\wedge i} & =\left\{\left(p, p_{0}\right): p \in X_{\text {sing }}, p_{0} \in X_{0}^{\wedge i}(p)\right\} \subset X_{\text {sing }} \times H_{0} .
\end{aligned}
$$

For every $Y \subset C^{n}$ we define

$$
\begin{aligned}
Y_{0}^{i}(p) & =\text { strict transform of } Y \text { under } \sigma_{p}^{i}, \\
Y_{0}^{i} & =\left\{\left(p, p_{0}\right): p \in X_{\text {sing }}, p_{0} \in Y_{0}^{i}(p)\right\} \subset X_{\text {sing }} \times C_{0}^{n}, \\
Y_{0}^{\vee i} & =\left\{\left(p, p_{0}\right): p \in X_{\text {sing }}, p_{0} \in Y_{0}^{i}(p) \cap H_{0}\right\} \subset X_{\text {sing }} \times H_{0} .
\end{aligned}
$$

For every $a \in N$ let

$$
\begin{aligned}
X_{1}^{i a}(p) & =\left(\psi^{a}\right)^{-1}\left(X_{0}^{i}(p)\right) \\
Y_{1}^{i a}(p) & =\left(\psi^{a}\right)^{-1}\left(Y_{0}^{i}(p)\right) \\
X_{1}^{i a} & =\left\{\left(p, p_{1}\right): p \in X_{\text {sing }}, p_{1} \in X_{1}^{i a}(p)\right\} \\
Y_{1}^{i a} & =\left\{\left(p, p_{1}\right): p \in X_{\text {sing }}, p_{1} \in Y_{1}^{i a}(p)\right\} \\
X_{1}^{\wedge i a}(p) & =X_{0}^{\wedge i}(p) \\
Y_{1}^{\text {via }}(p) & =Y_{0}^{\text {va }}(p)
\end{aligned}
$$


the two last sets are considered as subsets of $H_{1} ; H_{1}$ is just another copy of $H_{0}$.

By induction on $k$ we shall define subsets

$$
\begin{aligned}
X_{k}^{i a} & \subset X_{\text {sing }} \times H_{1} \times \ldots \times H_{k-1} \times \mathfrak{Ł}_{k}^{n}, \\
X_{k}^{\wedge i a} & \subset X_{\text {sing }} \times H_{1} \times \ldots \times H_{k-1} \times H_{k} ;
\end{aligned}
$$

they will be in the form

$$
\begin{array}{r}
X_{k}^{i a}=\left\{\left(p, p_{1}, \ldots, p_{k}\right):\left(p, p_{1}, \ldots, p_{k-1}\right) \in X_{k-1}^{\wedge i a},\right. \\
\left.\quad\left(p, p_{1}, \ldots, p_{k}\right) \in X_{k}^{i a}\left(p, p_{1}, \ldots, p_{k-1}\right)\right\}, \\
X_{k}^{\wedge i a}=\left\{\left(p, p_{1}, \ldots, p_{k}\right):\left(p, p_{1}, \ldots, p_{k-1}\right) \in X_{k-1}^{\wedge i a},\right. \\
\left.\left(p, p_{1}, \ldots, p_{k}\right) \in X_{k}^{\wedge i a}\left(p, p_{1}, \ldots, p_{k-1}\right)\right\} .
\end{array}
$$

It suffices to define $X_{k}^{i a}\left(p, p_{1}, \ldots, p_{k-1}\right)$ and $X_{k}^{\wedge i a}\left(p, p_{1}, \ldots, p_{k-1}\right)$; they are given by

$X_{k}^{i a}\left(p, p_{1}, \ldots, p_{k-1}\right)$ is the strict transform of

$$
\begin{gathered}
X_{k-1}^{i a}\left(p, p_{1}, \ldots, p_{k-2}\right) \text { under } \sigma_{p_{k-1}}: C_{k}^{n} \rightarrow C_{k-1}^{n}, \\
X_{k}^{\wedge i a}\left(p, p_{1}, \ldots, p_{k-1}\right)=N Z\left(X_{k}^{i a}\left(p, p_{1}, \ldots, p_{k-1}\right)\right) .
\end{gathered}
$$

For every $Y \subset C^{n}$ and $k \geqslant 1$ we define

$$
\begin{aligned}
Y_{k}^{i a} & =\left\{\left(p, p_{1}, \ldots, p_{k}\right):\left(p, p_{1}, \ldots, p_{k-1}\right) \in X_{k-1}^{\wedge i a}, p_{k} \in Y_{k}^{i a}\left(p, p_{1}, \ldots, p_{k-1}\right)\right\}, \\
Y_{k}^{\vee i a} & =\left\{\left(p, p_{1}, \ldots, p_{k}\right):\left(p, p_{1}, \ldots, p_{k-1}\right) \in X_{k-1}^{\wedge i a}, p_{k} \in Y_{k}^{\vee i a}\left(p, p_{1}, \ldots, p_{k-1}\right)\right\},
\end{aligned}
$$

where $Y_{k}^{i a}\left(p, p_{1}, \ldots, p_{k-1}\right)$ is the strict transform of $Y_{k-1}^{i a}\left(p, p_{1}, \ldots, p_{k-2}\right)$ under $\sigma_{p_{k-1}}$,

$$
Y_{k}^{\vee i a}\left(p, p_{1}, \ldots, p_{k-1}\right)=Y_{k}^{i a}\left(p, p_{1}, \ldots, p_{k-1}\right) \cap H_{k} .
$$

Our characterisation of sets $Y$ satisfying (*) is as follows.

Proposition. $Y$ satisties (*) if and only if $X_{k}^{\wedge i a} \subset Y_{k}^{\text {via }}$ for all $i, k, a$.

We shall give two applications of this proposition. We shall work only with (germs of) algebraic sets.

Let $K, N$ be given positive integers. Let $g_{i \alpha}$ be variables, $i=1, \ldots, K, \alpha$ $=\left(\alpha_{1}, \ldots, \alpha_{n}\right),|\alpha|=\alpha_{1}+\ldots+\alpha_{n} \leqslant N$. We consider $g_{i a}$ as coordinates in an affine space $G_{K N}$. Any point $g=\left(g_{i \alpha}\right) \in G_{K N}$ gives $K$ polynomials $g_{i}(x)$ $=\sum_{\alpha} g_{i \alpha} x^{a}$, and so we can define

$$
Y_{g}=\left\{x \in C^{n}: g_{\mathrm{i}}(x)=0 \text { for all } i=1, \ldots, K\right\} \text {. }
$$

Recall that the family of constructible sets in $\boldsymbol{C}^{n}$ is the Boolean algebra of subsets of $\boldsymbol{C}^{n}$ generated by all algebraic sets. 
Corollary 1. Let $X \subset C^{n}$ be an algebraic set of pure dimension d. Then, for every $K, N$, the sets

$$
\begin{aligned}
& G_{K N}(X)=\left\{g \in G_{K N}: Y_{g} \text { satisfies (*) in some neighbourhood of } 0\right\}, \\
& G_{K N}^{\prime}(X)=\left\{g \in G_{K N}(X): \operatorname{dim} Y_{g}<d\right\}
\end{aligned}
$$

are constructible (and, by [1], non-empty for sufficiently big $K, N$ ).

$G_{K N}(X), G_{K N}^{\prime}(X)$ are not always algebraic.

EXAmple. Let $X$ be a surface having 0 as an isolated singular point such that $C_{0}^{\prime}(X) \neq\{0\}$. By $[1]$, there exists a curve $Y$ satisfying $(*)$. By Lojasiewicz's inequality

$$
\left|P_{q}-P_{q^{\prime}}\right| \leqslant C\left|q-q^{\prime}\right| / \operatorname{dist}\left(\left\{q, q^{\prime}\right\}, 0\right)^{k} \quad \text { for all } q, q^{\prime} \in X \backslash\{0\},
$$

for some $k$, and therefore, as is easy to see, there exists an integer $p$ such that any curve $Y$ satisfies (*) provided that

$$
\operatorname{dist}\left(x, Y^{\prime}\right) \leqslant|x|^{p} \quad \text { for all } x \in Y \text {, sufficiently close to } 0 \text {. }
$$

Enlarging $Y$ if necessary, we can assume that it is given by $P_{1}=0, \ldots, P_{n-1}$ $=0$ and the ideal generated by the $P_{i}$ 's and the $(n-1) \times(n-1)$-minors of the matrix $\left(\partial P_{i} / \partial x_{j}\right)$ contains (for some $\left.m\right) m^{m}$, where $m=\left(x_{1}, \ldots, x_{n}\right)$. We take a coordinate system such that the $x_{n}$-axis is not contained in $C_{0}^{\prime}(X)$. Let $Y_{\varepsilon}$ be given by

$$
x_{1}^{N}=\varepsilon P_{1}(x), \ldots, x_{n-1}^{N}=\varepsilon P_{n-1}(x) .
$$

Then, if $N$ is big enough, we have, for every $\varepsilon \neq 0$,

$$
\operatorname{dist}\left(x, Y_{\varepsilon}\right) \leqslant|x|^{p} \quad \text { for } x \in Y,|x|<\delta_{\varepsilon} \text {, }
$$

where $\delta_{\varepsilon}>0$, and so every $Y_{\varepsilon}$ satisfies $(*)$ (for $\varepsilon \neq 0$ ). However $Y_{0}$ doesn't satisfy $(*)$, since $C_{0}(Y) \ngtr C_{0}^{\prime}(X)$.

Now let $X, Y \subset C^{n}$ be two algebraic sets, $X$ of pure dimension $d$. Let $L(X, Y)=\left\{p \in X_{\text {sing }}:(*)\right.$ is satisfied in a neighbourhood of $p$, with a constant $C$ depending on $p_{l}^{\text {? }}$,

$N L(X, Y)=X_{\text {sing }} \backslash L(X, Y)$.

Corollary 2. NL $(X, Y)$ is algebraic.

In the sequel the letter $C$ will denote different constants.

\section{Preliminaries}

We consider two copies $C_{x}^{n}, C_{y}^{n}$ of $C^{n}$ with coordinates $x_{1}, \ldots, x_{n}$ and $y_{1}, \ldots, y_{n}$ respectively. Let $H_{x}=\left\{x_{1}=0\right\} \subset C_{x}^{n}, H_{y}=\left\{y_{1}=0\right\} \subset C_{y}^{n}$. We 
shall list some obvious properties of the maps $\sigma, \psi: C_{y}^{n} \rightarrow C_{x}^{n}$ given by

$$
\begin{aligned}
\sigma\left(y_{1}, \ldots, y_{n}\right) & =\left(y_{1}, y_{1} y_{2}, \ldots, y_{1} y_{n}\right)(\sigma \text {-process }), \\
\psi\left(y_{1}, \ldots, y_{n}\right) & =\left(y_{1}^{a}, y_{2}, \ldots, y_{n}\right),
\end{aligned}
$$

where $a$ is a given positive integer. Let $f$ be either $\sigma$ or $\psi$.

For $q \in C_{x}^{n}$ (resp. $\left.\tilde{q} \in C_{y}^{n}\right)$ let $H_{x, q}$ (resp. $H_{y, \tilde{q}}$ ) be the hyperplane parallel to $H_{x}$ (resp. $H_{y}$ ) passing through $q$ (resp. $\tilde{q}$ ).

Let $\tilde{q}_{1}, \tilde{q}_{2} \in \boldsymbol{C}_{y}^{n}$ and $q_{1}=f\left(\tilde{q}_{1}\right), q_{2}=f\left(\tilde{q}_{2}\right)$. Let

$$
\tilde{\Pi}_{i} \subset T_{\tilde{q}_{i}} C_{y}^{n} \cap H_{y, \tilde{q}_{i}}, \quad i=1,2,
$$

be two linear subspaces and let $\Pi_{i} \subset T_{q_{i}} C_{x}^{n}$ be their images under $d f$. Since $C_{x}^{n}, C_{y}^{n}$ are linear spaces, we can speak about the angles $\Varangle\left(\tilde{\Pi}_{1}, \tilde{\Pi}_{2}\right)$, $\Varangle\left(\Pi_{1}, \Pi_{2}\right)$;

$$
\Varangle\left(\tilde{\Pi}_{1}, \tilde{\Pi}_{2}\right)=\Varangle\left(\Pi_{1}, \Pi_{2}\right) .
$$

This is obvious, since

$$
\left.\left.\sum_{j} a_{j}\left(\partial / \partial y_{j}\right)\right|_{\tilde{q}_{i}} \in \tilde{\Pi}_{i} \Leftrightarrow \sum_{j} a_{j}\left(\partial / \partial x_{j}\right)\right|_{q_{i}} \in \Pi_{i} \quad \text { for } i=1,2 .
$$
define

Let $X \subset C_{x}^{n}\left(\right.$ resp. $\left.\tilde{X} \subset C_{y}^{n}\right)$ be analytic sets. For $q \in X_{\text {reg }}$ (resp. $\tilde{q} \in \tilde{X}_{\text {reg }}$ ) we

$$
T_{q}^{0} X=T_{q} X \cap H_{x, q} \quad\left(\text { resp. } T_{\tilde{q}}^{0} \tilde{X}=T_{\tilde{q}} \tilde{X} \cap H_{y, \tilde{q}}\right)
$$

Let

$$
P_{q}^{0}: T_{q} C_{x}^{n} \rightarrow T_{q}^{0} X, \quad P_{\tilde{q}}^{0}: T_{\tilde{q}} C_{y}^{n} \rightarrow T_{\tilde{q}}^{0} \tilde{X}
$$

be the orthogonal projections and

$$
P_{q}^{0 \perp}=I-P_{q}^{0}, \quad P_{\tilde{q}}^{0 \perp}=I-P_{\tilde{q}}^{0} .
$$

Let $X \subset C_{x}^{n}$ be a given analytic set and take for $\tilde{X}$ the strict transform of $X$ under $\sigma$ if $f=\sigma$ and $\psi^{-1}(X)$ if $f=\psi$. Let $\tilde{q}_{1}, \tilde{q}_{2} \in \tilde{X}_{\mathrm{reg}} \backslash H_{y}, q_{i}=f\left(\tilde{q}_{i}\right)$ for $i=1,2$. Then (5) gives

$$
\left|P_{\bar{q}_{1}}^{0 \perp} P_{\tilde{q}_{2}}^{0}\right|=\left|P_{q_{1}}^{0 \perp} P_{q_{2}}^{0}\right| .
$$

For every $q \in C_{x}^{n}$ (resp. $\left.\tilde{q} \in C_{y}^{n}\right)$ we put

(10) $d^{0}(q, X)=\operatorname{dist}\left(q, X \cap H_{x, q}\right) \quad\left(\right.$ resp. $\left.d^{0}(\tilde{q}, \tilde{X})=\operatorname{dist}\left(\tilde{q}, \tilde{X} \cap H_{y, \tilde{q}}\right)\right)$.

Let $\tilde{q} \in C_{y}^{n}$ and $q=f(\tilde{q}) \in C_{x}^{n}$; then we have

$$
d^{0}(q, X)= \begin{cases}d^{0}(\tilde{q}, \tilde{X}), & \text { if } f=\psi, \\ q_{1} d^{0}(\tilde{q}, \tilde{X}), & \text { if } f=\sigma,\end{cases}
$$

where $q_{1}$ is the $x_{1}$-coordinate of $q$. 
If $q(t)$ is a germ of a curve at $0 \in C_{x}^{n}$ such that

$$
v=\lim _{i \rightarrow 0} \dot{q}(t) /|\dot{q}(t)| \in C_{0}(X),
$$

but $\Varangle\left(v, H_{x}\right)>\varepsilon$, then there exists a $C$, depending on $\varepsilon$, such that

$$
d^{0}(q(t), X) \leqslant C \operatorname{dist}(q(t), X) .
$$

We shall also use two trivial observations from elementary geometry. Let $H=H^{n-1} \subset C^{n}$ be a hyperplane and $\Pi_{1}^{0}, \Pi_{2}^{0}$ two linear subspace of $H$, of the same dimension. Let $v_{1}, v_{2}$ be two unit vectors in $C^{n}$ such that

$$
\Varangle\left(H, v_{i}\right) \geqslant \beta>0 \quad \text { for } i=1,2,\left|v_{1}-v_{2}\right| \leqslant \alpha .
$$

Put $\Pi_{i}=\Pi_{i}^{0} \oplus C v_{i}$ and let $P_{i}$ (resp. $P_{i}^{0}$ ) be the orthogonal projection of $C^{n}$ onto $\Pi_{i}$ (resp. $\left.\Pi_{i}^{0}\right), P_{i}^{\perp}=I-P_{i}, P_{i}^{0 \perp}=I-P_{i}^{0}$. Then

$$
\begin{aligned}
\left|P_{2}^{0 \perp} P_{1}^{0}\right| & \leqslant\left|P_{2}^{\perp} P_{1}\right|(1+(1 / \sin \beta)), \\
\left|P_{2}^{\perp} P_{1}\right| & \leqslant\left|P_{2}^{0 \perp} P_{1}^{0}\right|(1+(1 / \sin \beta))+(\alpha / \sin \beta) .
\end{aligned}
$$

Let $\pi: \boldsymbol{C}^{n} \rightarrow \boldsymbol{C}^{d}$ be the standard projection, $\pi\left(x_{1}, \ldots, x_{n}\right)=\left(x_{1}, \ldots, x_{d}\right)$ and let $T, T^{\prime}$ be two $d$-dimensional planes in $C^{n}$ such that $\Varangle(T, k e r \pi)>\varepsilon$, $\Varangle\left(T^{\prime}, \operatorname{ker} \pi\right)>\varepsilon$. If $w_{k}, w_{k}^{\prime}$ are liftings of $\partial / \partial x_{k}$ to $T, T^{\prime}$ respectively $(k \leqslant d)$, then, for some $C, C^{\prime}$, depending on $\varepsilon$,

$$
C \max \Varangle\left(w_{k}, w_{k}^{\prime}\right) \leqslant \Varangle\left(T, T^{\prime}\right) \leqslant C^{\prime} \max \Varangle\left(w_{k}, w_{k}^{\prime}\right) .
$$

\section{A characterisation of $Z$-points}

Let $X \subset C^{n}$ be an analytic set of pure dimension $d$ and $H=\left\{x_{1}=0\right\} \subset C^{n}$. $T_{q}^{0} X, P_{q}^{0}, P_{q}^{0 \perp}$ are defined (for $q \in X_{\mathrm{reg}}$ ) as in (7) and (8).

Lemma 1. A point $p \in X \cap H$ is a $Z$-point of $X$ if and only if there exists a $C$, depending on $p$, such that

$$
\left|P_{q_{1}}^{0 \perp} P_{q_{2}}^{0}\right| \leqslant C\left|q_{1}-q_{2}\right|
$$

for all $q_{1}, q_{2} \in X_{\mathrm{reg}} \backslash H$, having the same $x_{1}$-coordinate, lying in a suitable neighbourhood of $p$.

Proof of the "if" part. Let $p$ be a $Z$-point. We choose axes $x_{d+1}, \ldots, x_{n}$ in $H$ so that the coordinates of points of $X$ satisfy (2) and (3). Then if $q \in X$ has coordinates

$$
\left(x_{1}, \ldots, x_{d}, \psi_{d+1, \alpha_{d}+1}\left(x_{1}^{1 / r}, \ldots, x_{d}\right), \ldots, \psi_{n, \alpha_{n}}\left(x_{1}^{1 / r}, \ldots, x_{d}\right)\right),
$$

then $T_{q}^{0} X$ is spanned by

$$
w_{k}(q)=\left(\partial / \partial x_{k}\right)+\sum_{i=d+1}^{n}\left[\left(\partial / \partial x_{k}\right) \psi_{i, a_{i}}\right] \partial / \partial x_{i},
$$


$1<k \leqslant d$. It is easy to prove (the details are in [1]) that $\left|w_{k}(q)\right| \leqslant C$, $\left|w_{k}(q)-w_{k}\left(q^{\prime}\right)\right| \leqslant C\left|q-q^{\prime}\right|$ for all $k$ and for every $q^{\prime} \in X_{\text {reg }} \backslash H$ having the same $x_{1}$-coordinate as $q$. This, together with (14), implies (15).

For the "only if" part we need a lemma.

Lemma 2. Let $\Gamma \subset C^{n}$ be a germ of a curve at $p$, singular at $p$, and let $l \subset C^{n}$ be a line. Assume that the orthogonal projection $\pi_{l}: C^{n} \rightarrow l$ induces an isomorphism $T_{q} \Gamma \rightarrow T_{\pi_{r}(q)}$ l for every $q \neq p$ and the norm of its inverse is $\leqslant C, C$ independent of $q$. Then there exist sequences of points $q_{v}, q_{v}^{\prime} \in \Gamma_{\text {reg }}$ such that $q_{v}^{\prime} \neq q_{v}, q_{v}, q_{v}^{\prime} \rightarrow p$, and

$$
\Varangle\left(T_{q_{v}} \Gamma, T_{q_{v}^{\prime}} \Gamma\right) /\left|q_{v}-q_{\nu}^{\prime}\right| \rightarrow \infty .
$$

Proof. We can assume that $p=0$ and $l$ is the $x_{1}$-axis. Consider the case $n=2$. Assume first that $\Gamma$ contains a component $\Gamma_{0}$, singular at the origin. Then $\Gamma_{0}$ can be described by

$$
x_{1}=t^{r}, \quad x_{2}=\lambda(t), \quad r \geqslant 2, \lambda \text { analytic. }
$$

For every $r$-th root of unity $\varepsilon \neq 1$ we have $\lambda(\varepsilon t) \neq \lambda(t)$ for all $t \neq 0$, so

$$
\lambda(\varepsilon t)-\lambda(t)=t^{k} u(t), \quad u(0) \neq 0,
$$

and thus $\lambda^{\prime}(s t)-\lambda^{\prime}(t)$ is of order $t^{k-1}$. It is easy to calculate that if $q$ corresponds to $t \neq 0$ and $q^{\prime}$ to $\varepsilon t$, then $\left|q-q^{\prime}\right|$ is of order $|t|^{k}$, while $\Varangle\left(T_{q} \Gamma, T_{q}, \Gamma\right)$ is of order $|t|^{k-r}$.

In the other ease $\Gamma$ contains two nonsingular components $\Gamma_{1}, \Gamma_{2}$, intersecting at 0 , given by

$$
\Gamma_{1}: x_{2}=\lambda\left(x_{1}\right), \quad \Gamma_{2}: x_{2}=\mu\left(x_{1}\right), \quad \lambda, \mu \text { analytic. }
$$

Clearly $\lambda(0)=\mu(0)=0$ and $\lambda\left(x_{1}\right) \neq \mu\left(x_{1}\right)$ for $x_{1} \neq 0$. We take $q$ lying on $\Gamma_{1}$ and $q^{\prime}$ on $\Gamma_{2}$ and repeat the reasoning.

If $n>2$, we take a linear projection $\pi: C^{n} \rightarrow P$ on a plane $P$ containing $l$ such that $\pi \mid \Gamma$ is proper and, for some $C$,

$$
\left|q-q^{\prime}\right| \leqslant C\left|\pi(q)-\pi\left(q^{\prime}\right)\right| \quad \text { for all } q, q \in \Gamma_{\text {reg }} \text {, close to } 0 .
$$

Necessarily $\pi(0)$ is a singular point of $\pi(\Gamma)$. Let $\tilde{q}_{v}, \tilde{q}_{v}^{\prime} \in \pi(\Gamma)_{\text {reg }}$ satisfy the conclusion of the lemma for $\pi(\Gamma)$ and we take for $q_{v}, q_{v}^{\prime}$ any points in $\Gamma_{\text {reg }}$ projecting into $\tilde{q}_{v}, \tilde{q}_{v}^{\prime}$.

Returning to the proof of Lemma 1 we take a point $p \in X \cap H$ such that (15) is satisfied in a neighbourhood of $p$. Let $p_{t}$ be a germ of an analytic map $(C, 0) \rightarrow\left(C^{n}, p\right)$ such that $p_{t} \in X_{\text {rep }} \backslash H$ for $t \neq 0$. There exists an open and dense set $D$ of projections $\boldsymbol{C}^{n} \rightarrow C^{d}$, parallel to $H$, such that for every $\pi \in D$ we have: $\pi: X \rightarrow C^{d}$ is proper and for some $C=C(\pi)$ and every $t \neq 0$, $d \pi\left(p_{t}\right): T_{p_{t}}^{0} X \rightarrow T_{\pi\left(p_{t}\right)}^{0} C^{d}$ is an isomorphism and the norm of its inverse is bounded by $C$. 
Fix a $\pi \in D$; after a translation and a coordinate change in $H$ we can assume that $p=0, \pi\left(x_{1}, \ldots, x_{n}\right)=\left(x_{1}, \ldots, x_{d}\right)$.

$1^{\text {o }}$ There exists a $C^{\prime}=C^{\prime}(\pi)$ such that for every $q \in X_{\text {reg }} \backslash H$, sufficiently close to $p$,

$$
d \pi(q): T_{q}^{0} X \rightarrow T_{\pi(q)}^{0} C^{d}
$$

is an isomorphism and the norm of its inverse is bounded by $C^{\prime}$. For otherwise, using the curve selection lemma for real semianalytic sets, we prove that there exists a real-analytic map $q(r)$ such that $q(r) \in X_{\text {reg }} \backslash H$ for $r \neq 0, q(0)=0$ and the norm of the inverse to $d \pi(q(r))$ tends to $\infty(r \in R)$. Let $q(t)(t \in C)$ be the complexification of $q(r)$. We reparametrise $q(t)$ and $p(t)$ so that the $x_{1}$-coordinates of $q(t)$ and $p(t)$ coincide and we get a contradiction with (15).

$2^{\circ} \quad X_{\text {sing }} \subset H$. For suppose it is not so. Then $\operatorname{dim} \overline{X_{\text {sing }} \backslash H}=d-1$. Let $W=\pi\left(\overline{X_{\text {sing }} \backslash H}\right)$; clearly $\operatorname{dim} W=d-1$. A general line $l$ in $C^{d}$, parallel to $\left\{x_{1}=0\right\}$, intersects $W$ transversally; its lifting $\Gamma$ to $X$ has singular points. Applying to $\Gamma$ Lemma 2 we get sequences of points $q_{v}, q_{v}^{\prime} \in \Gamma_{\text {reg }}$ such that $\Varangle\left(T_{q_{v}} \Gamma, T_{q_{v}^{\prime}} \Gamma\right) /\left|q_{v}-q_{v}^{\prime}\right| \rightarrow \infty$. Now, using (14), we get a contradiction with (15).

$3^{\circ}$ Let $x^{\prime}=\left(x_{2}, \ldots, x_{d}\right), y=\left(x_{d+1}, \ldots, x_{n}\right)$. It follows from $2^{\circ}$ that there exists finitely many $C^{n-d}$-valued analytic functions $\varphi_{a}\left(t, x^{\prime}\right), \varphi_{a}$ $=\left(\varphi_{\alpha, d+1}, \ldots, \varphi_{a, n}\right)$ and an integer $r$ such that if $\left(x_{1}, x^{\prime}, y\right) \in X$, then, for some $\alpha$,

$$
y=\varphi_{a}\left(x_{1}^{1 / r}, x^{\prime}\right) .
$$

For any $\alpha$ and any $r-$ th root of unity $\varepsilon$ we put

$$
\psi\left(t, x^{\prime}\right)=\varphi_{a}\left(\varepsilon t, x^{\prime}\right)-\varphi_{a}\left(t, x^{\prime}\right)=\left(\psi_{d+1}\left(t, x^{\prime}\right), \ldots, \psi_{n}\left(t, x^{\prime}\right)\right) .
$$

For these $\psi$ that don't vanish identically let (for a generic $x^{\prime}$ ) ord $\psi_{i}=s_{i}$ (at $t$ $=0$ ); after a permutation of $x_{d+1}, \ldots, x_{n}$ we can assume for simplicity that $s_{d+1}=\ldots=s_{d+k}=s$ and $s_{i}>s$ for $i>d+k$. Thus

$$
\psi_{i}=t^{s_{i}} \tilde{\psi}_{i}\left(t, x^{\prime}\right), \quad \tilde{\psi}_{i}\left(0, x^{\prime}\right) \quad \text { not identically } 0 \text {. }
$$

We shall show that at least one of the $\tilde{\psi}_{i}$ for $i \leqslant d+k$ is $\neq 0$ at $x^{\prime}=0=\pi(p)$.

Suppose that $\tilde{\psi}_{i}(0,0)=0$ for all $i \leqslant d+k$. Then

$$
\begin{aligned}
& \psi_{i}\left(t, x^{\prime}\right)=t^{s} \psi_{i}^{*}\left(x^{\prime}\right)+O\left(t^{s+1}\right), \quad \psi_{i}^{*}(0)=0, \quad i \leqslant d+k, \\
& \psi_{i}\left(t, x^{\prime}\right)=O\left(t^{s+1}\right), \quad i>d+k .
\end{aligned}
$$

For $t, x^{\prime}$ unprecised for the moment let

$$
q=\left(t^{r}, x^{\prime}, \varphi_{a}\left(t, x^{\prime}\right)\right), \quad q^{\prime}=\left(t^{r}, x^{\prime}, \varphi_{a}\left(\varepsilon t, x^{\prime}\right)\right) \in X .
$$


Then $\left|q-q^{\prime}\right|=\left|\psi\left(t, x^{\prime}\right)\right|$ and it follows from $1^{\circ}$, (14) that

$$
\Varangle\left(T_{q}^{0} X, T_{q^{\prime}}^{0} X\right) \text { is of order } \max _{2 \leqslant j \leqslant d}\left|\partial \psi\left(t, x^{\prime}\right) / \partial x_{j}\right|,
$$

so (15) implies that

$$
\left|\hat{o}_{j} \psi(t, x)\right| \leqslant C|\psi(t, x)| \quad \text { for } 2 \leqslant j \leqslant d,
$$

where $\partial_{j}=\partial / \partial x_{j}$. For $2 \leqslant j \leqslant d$ we have

$$
\begin{aligned}
& \partial_{j} \psi_{i}\left(t, x^{\prime}\right)=t^{s} \partial_{j} \psi_{i}^{*}\left(x^{\prime}\right)+O\left(t^{s+1}\right), \quad i \leqslant d+k, \\
& \partial_{j} \psi_{i}\left(t, x^{\prime}\right)=O\left(t^{s+1}\right), \quad i>d+k .
\end{aligned}
$$
then

Let $\psi_{i}^{0}\left(x^{\prime}\right)$ be the homogenous part of $\psi_{i}^{*}$, of degree, say, $m_{i}(i \leqslant d+k)$;

$$
\psi_{i}^{*}\left(x^{\prime}\right)=\psi_{i}^{0}\left(x^{\prime}\right)+O\left(\left|x^{\prime}\right|^{m_{i}+1}\right) .
$$

Let $\Omega$ be an open cone in the $x^{\prime}$-space, with vertex at 0 , disjoint with all the cones $\psi_{i}^{0}\left(x^{\prime}\right)=0$. Let $m=\min m_{i}$; again we can assume that $m_{i}=m$ for $i \leqslant d+l, m_{i}>m$ for $i>d+l$, where $l$ is some number $\leqslant k$. After a linear change among $x_{2}, \ldots, x_{d}$ we can assume that for all $i \leqslant d+l$

$$
\left|\partial_{2} \psi_{i}^{0}\left(x^{\prime}\right)\right| \geqslant C\left|x^{\prime}\right|^{m-1} \quad \text { for } x^{\prime} \in \Omega
$$

clearly for all $x^{\prime}$

$$
\left|\psi_{i}^{*}\left(x^{\prime}\right)\right| \leqslant C\left|x^{\prime}\right|^{m}
$$

Now take in (16) $x^{\prime} \in \Omega,|t|=\left|x^{\prime}\right|^{m}$. Then

$$
\begin{aligned}
& \left|\psi_{i}\left(t, x^{\prime}\right)\right| \leqslant|t|^{s}\left|\psi_{i}^{*}\left(x^{\prime}\right)\right|+\left|x^{\prime}\right|^{m(s+1)} \leqslant C\left|x^{\prime}\right|^{m(s+1)}, \quad i \leqslant d+k, \\
& \left|\psi_{i}\left(t, x^{\prime}\right)\right| \leqslant C|t|^{s+1} \leqslant C\left|x^{\prime}\right|^{m(s+1)}, \quad i>d+k,
\end{aligned}
$$

so

$$
\left|\psi\left(t, x^{\prime}\right)\right| \leqslant C\left|x^{\prime}\right|^{m(s+1)}
$$

But for $i \leqslant d+l$

$$
\begin{aligned}
\left|\partial_{2} \psi_{i}\left(t, x^{\prime}\right)\right| & \geqslant|t|^{s}\left|\hat{\partial}_{2} \psi_{i}^{*}\left(x^{\prime}\right)\right|-O\left(|t|^{s+1}\right) \\
& \geqslant C|t|^{s}\left|x^{\prime}\right|^{m-1}-C|t|^{s}\left|x^{\prime}\right|^{m}-C\left|x^{\prime}\right|^{m(s+1)} \geqslant C\left|x^{\prime}\right|^{(s+1)-1}
\end{aligned}
$$

and we have a contradiction with (17).

$4^{\circ}$ After a linear change in the $x_{d+1}, \ldots, x_{n}$-coordinates we can assume that for all $\alpha, \varepsilon$, and $i$, either $\psi_{i}$ is identically 0 or $\psi_{i}(0,0) \neq 0$. This shows that the set of $\pi \in D$ for which the latter condition holds, is open and dense. But this is equivalent to the definition of a $Z$-point, so $p \in Z(X)$. 


\section{Proof of the proposition}

Let $X \subset C^{n}$ be analytic, $\operatorname{dim} X=d$.

Lemma 3. Let $\Gamma$ be a germ at $p \in X$ of a curve such that $\Gamma \backslash\{p\} \subset X_{\mathrm{reg}}$. Then the $C^{\infty}$-function

$$
\Gamma_{\text {reg }} \ni q \mapsto P_{q} \in C^{n^{2}}
$$

(where, of course, $P_{q}: C^{n} \rightarrow T_{q} X$ ) satisfies, for some $C$,

$$
\left|D P_{q}\right| \leqslant C /|q-p|
$$

(even $\left|D P_{q}\right| \leqslant C /|q-p|^{\alpha}$ for some $\alpha<1$, but we don't need that).

Proof. Assume that $p=0$. Let $\pi: C^{n} \rightarrow C^{d}$ be a projection such that for all $q \in \Gamma \backslash\{0\} d \pi(q): T_{q} X \rightarrow T_{\pi(q)} C^{d}$ is an isomorphism and the norm of its inverse is bounded by some $C$. After a coordinate change in $C^{d}$ we can assume that $\Gamma$ is given by $x_{i}=\lambda_{i}\left(x^{1 / r}\right), i=2, \ldots, n, \lambda_{i}$ analytic, ord $\lambda_{i} \geqslant r$. Let $w_{1}(q), \ldots, w_{d}(q)$ be liftings of $\partial / \partial x_{1}, \ldots, \partial / \partial x_{d}$ to vectors in $T_{q} X, q \in \Gamma_{\text {reg }}$; then $w_{j}$ are analytic in $x_{1}^{1 / s}$ for some $s$; since they are bounded, ord $w_{j} \geqslant s$. Thus $\left|D w_{j}\right| \leqslant C /\left|x_{1}\right|^{1-(1 / s)}$.

Now let $\Gamma$ be again a germ of a curve at $p$ (=0 for simplicity), $\Gamma \backslash\{p\} \subset X_{\text {reg }}$; let $\Gamma$ be given by $x_{i}=\lambda_{i}\left(x_{1}^{1 / r}\right)$, ord $\lambda_{i} \geqslant r$.

Lemma 4. For every $q \in \Gamma \backslash\{0\}$ and any number $a \in C,|a| \neq 0$ and small enough, there exists a $q^{\prime} \in \Gamma \backslash\{0\}$ having $a$ as its $x_{1}$-coordinate such that

$$
\left|P_{q}^{\perp} P_{q^{\prime}}\right| \leqslant C\left|q-q^{\prime}\right| / \min \left(\left|q_{1}\right|,|a|\right),
$$

where $q_{1}$ is the $x_{1}$-coordinate of $q$ and $C$ is independent of $q, a$.

Proof. In the $x_{1}$-axis we join $q_{1}$ and $a$ by an arc $L$ of length $\leqslant 2 \pi\left|q_{1}-a\right|$ such that for every $t \in L$

$$
|t| \geqslant \min \left(\left|q_{1}\right|,|a|\right) .
$$

We lift $L$ to a real curve in $\Gamma_{\text {reg }}$, starting at $q$. If $q^{\prime}$ is its end, then, by Lemma 3,

$$
\left|P_{q}^{\perp} P_{q^{\prime}}\right| \leqslant C\left|q_{1}-a\right| / \min \left(\left|q_{1}\right|,|a|\right) \leqslant C\left|q-q^{\prime}\right| / \min \left(\left|q_{1}\right|,|a|\right) .
$$

Lemma 4, together with (13), implies the following lemma.

Lemma 5. Let $\Gamma_{1}, \Gamma_{2}$ be germs of curves at $p \in X, \Gamma_{i} \backslash\{p\} \subset X_{\text {reg }}$, such that the angles between the tangent vectors to $\Gamma_{i}$ and $\partial / \partial x_{1}$ are $<(\pi / 2)-\alpha$, $\alpha>0$. Let $H=\left\{x_{1}=0\right\}$ and let $Y \subset C^{n}$ be any analytic set. Then the following conditions are equivalent:

$$
\begin{array}{ll}
1^{\circ} & \left|P_{q_{1}}^{1} P_{q_{2}}\right| \leqslant C\left|q_{1}-q_{2}\right| / \operatorname{dist}\left(\left\{q_{1}, q_{2}\right\}, Y\right) \text { for all } q_{i} \in \Gamma_{i} \backslash\{p\} ; \\
2^{\circ} & \left|P_{q_{1}}^{0 \perp} P_{q_{2}}^{0}\right| \leqslant C\left|q_{1}-q_{2}\right| / \operatorname{dist}\left(\left\{q_{1}, q_{2}\right\}, Y\right) \text { for all } q_{i} \in \Gamma_{i} \backslash\{p\}, \quad i=1,2,
\end{array}
$$
such that $q_{1}, q_{2}$ have the same $x_{1}$-coordinate. 
We can now prove the proposition. We shall use the same symbols $P^{0}$, $P^{0 \perp}$ for projections onto $T^{0} X_{k}^{i a}, T^{0 \perp} X_{k}^{i a}$ for various $i, a, k$.

$1^{\circ}$ Assume that $Y_{k}^{v i a} \doteq X_{k}^{\wedge i a}$ for some $k, i$, a ; we shall show that $(*)$ doesn't hold. We assume that the smallest $k$ with the latter property is $>1$ (the case $k=1$, or, which is the same, $k=0$, we leave to the reader). Thus, for every $p \in X_{\text {sing }}$ we have $C_{p}^{\prime}(X) \subset C_{p}(Y)$.

Let $\tilde{p}=\left(p, p_{1}, \ldots, p_{k-1}\right) \in N Z\left(X_{k-1}^{i a}\right) \backslash Y_{k-1}^{\vee i a}$. By Lemma 1 and the curve selection lemma there exist germs of analytic maps $\tilde{q}_{1}(t), \tilde{q}_{2}(t)$ such that $\tilde{q}_{1}(0)=\tilde{q}_{2}(0)=\tilde{p}, \tilde{q}_{1}(t), \tilde{q}_{2}(t) \in X_{k-1, \text { reg }}^{i a}$ for $t \neq 0$, the $x_{1}$-coordinates of $\tilde{q}_{1}(t)$, $\tilde{q}_{2}(t)$ coincide, and

$$
\left|P_{\tilde{q}_{1}(t)}^{0 \perp} P_{\bar{q}_{2}(t)}^{0}\right| /\left|\tilde{q}_{1}(t)-\tilde{q}_{2}(t)\right| \text { is unbounded. }
$$

Let $q_{1}(t), q_{2}(t)$ be the images of $\tilde{q}_{1}(t), \tilde{q}_{2}(t)$ under $\sigma_{p}^{i} \psi^{a} \sigma_{p_{1}} \ldots \sigma_{p_{k-1}}$; then, by (9), (11)

$$
\left|P_{q_{1}(t)}^{0 \perp} P_{q_{2}(t)}^{0}\right| d^{0}\left(\left\{q_{1}(t), q_{2}(t)\right\}, Y\right) /\left|q_{1}(t)-q_{2}(t)\right| \text { is unbounded. }
$$

Clearly, $q_{1}(t), q_{2}(t)$ are tangent at $p$ to $C_{p}^{\prime}(X) \subset C_{p}(Y)$ and $q_{1}(t)$ and $q_{2}(t)$ have the same $x_{i}$-coordinates; further

$$
\lim _{i \rightarrow 0}\left(\dot{q}_{j}(t) /\left|\dot{q}_{j}(t)\right|\right) \notin\left\{x_{i}=0\right\} \quad \text { for } j=i \cdot 1,2 .
$$

Now (12) and (13) imply that

$$
\left|P_{q_{1}(t)}^{\perp} P_{q_{2}(t)}\right| \operatorname{dist}\left(\left\{q_{1}(t), q_{2}(t)\right\}, Y\right) /\left|q_{1}(t)-q_{2}(t)\right| \text { is unbounded, }
$$

so (*) is not satisfied.

$2^{\circ}$ Assume that $X_{k}^{\wedge i a} \subset Y_{k}^{v i a}$ for all $i, a, k$, but (*) is not satisfied. Then, by the curve selection lemma, there exist germs of analytic maps $q_{1}(t), q_{2}(t)$ such that $q_{1}(0)=q_{2}(0)=p \in X_{\text {sing }}, q_{1}(t), q_{2}(t) \in X_{\text {reg }}$ for $t \neq 0$ and

$$
\left|P_{q_{1}(t)}^{\perp} P_{q_{2}(t)}\right| \operatorname{dist}\left(\left\{q_{1}(t), q_{2}(t)\right\}, Y\right) /\left|q_{1}(t)-q_{2}(t)\right| \text { is unbounded ([1]). }
$$

Select one of the coordinate axes, $x_{i}$, such that, for some $\alpha>0$,

$$
\Varangle\left(\lim _{t \rightarrow 0}\left(\dot{q}_{j}(t) /\left|\dot{q}_{j}(t)\right|\right),\left\{x_{i}=0\right\}\right) \leqslant \frac{1}{2} \pi-\alpha .
$$

By Lemma 5 we can assume that $q_{1}(t), q_{2}(t)$ have the same $x_{i}$-coordinate. Let $q_{1}^{*}(t), q_{2}^{*}(t)$ be the liftings of $q_{1}(t), q_{2}(t)$ via $\sigma_{p}^{j}$; then

$$
\left|P_{q_{1}(t)}^{0+1} P_{q_{2}^{*}(t)}^{0}\right| d^{0}\left(\left\{q^{*}(t), q_{2}^{*}(t)\right\}, Y_{0}^{i}\right) /\left|q_{1}^{*}(t)-q_{2}^{*}(t)\right| \text { is unbounded. }
$$

We choose $a$ so that $\left(\psi^{a}\right)^{-1}\left(q_{j}^{*}(t)\right)$ are sums of smooth curves $\Gamma_{j \beta}$ given by

$$
\Gamma_{j \beta}: x_{s}=\varphi_{j \beta, s}\left(x_{1}\right), \quad s=2, \ldots, n .
$$

By (9), (11), (18) remains unchanged if we pass from $q_{j}^{*}(t)$ to $\left(\psi^{0}\right)^{-1}\left(q_{j}^{*}(t)\right)$ and 
from $X_{0}^{i}, Y_{0}^{i}$ to $X_{1}^{i a}, Y_{1}^{i a}$; further it remains unchanged after liftings via the $\sigma$ processes. But after a finite number of such liftings the strict transforms of $\Gamma_{j \beta}$ become disjoint and we get a contradiction.

\section{Constructibility of $X_{k}^{\wedge i a}$ and $Y_{k}^{v i a}$}

First we introduce some notation. If

$$
F(t)=b_{0}+b_{1} t+\ldots+b_{n-1} t^{n-1}+t^{n},
$$

we define $([1])$

$$
\Delta_{i}^{F}=\sum_{a_{1}, \ldots, a_{i}}^{*} \prod_{r, s}^{*}\left(t_{r}-t_{s}\right)
$$

where $\sum^{*}$ denotes the summation over all $\alpha_{k}$ such that $\alpha_{k} \neq \alpha_{j}$ for $k \neq j$, and $\prod^{*}$ the product over all $r, s$ such that $r \neq s$ and $r, s \neq \alpha_{j}$ for all $j$. The $t_{r}$ are of course all the roots of $F$. We consider $\Delta_{i}^{F}$ as polynomials in $b_{0}, \ldots, b_{n-1}$. Thus $\Delta_{0}^{F}$ is the discriminant of $F$ and $F$ has less than $n-k$ distinct roots if and only if $\Delta_{i}^{F}=0$ for all $i \leqslant k$.

If $F(t)=a_{0}+a_{1} t+\ldots+a_{n} t^{n}$, then we put $G=b_{0}+b_{l} t+\ldots+b_{n-1} t^{n-1}$ $+t^{n}$, where $b_{i}=a_{i} / a_{n}$, and

$$
\Delta_{i}^{F}\left(a_{0}, \ldots, a_{n}\right)=a_{n}^{k(i)} \Delta_{i}^{G}\left(b_{0}, \ldots, b_{n-1}\right),
$$

where $k(i)$ is the smallest number such that $\Delta_{i}^{F}$ is a polynomial.

Lemma 6. Let $S \subset C^{p}$ be algebraic and $X \subset S \times C^{n}$ algebraic. Let $\pi: S$ $\times C^{n} \rightarrow S$ be the standard projection, $H=\left\{x_{1}=0\right\} \subset C^{n}$; assume that all the fibers $X_{s}=\pi^{-1}(s) \cap X \subset\{s\} \times C^{n} \approx C^{n}$ are of pure dimension $d$ and $\operatorname{dim} X_{s} \cap H<d$. Then there exists an algebraic set $S_{0} \varsubsetneqq S$ and an algebraic set $Z \subset S \times C^{n}$ such that $Z_{s}=N Z\left(X_{s}\right)$ for all $s \in S \backslash S_{0}$, where $Z_{s}=Z \cap \pi^{-1}(s)$.

Proof. Assume first that $d=n-1$. There exists a polynomial $F(s, x)$ $\left(s \in C^{p}, x \in C^{n}\right)$ and an algebraic set $S_{1} \mp S$ such that

$$
X_{s}=\{x: F(s, x)=0\} \quad \text { for all } s \in S \backslash S_{1} .
$$

For every $\xi \in H$ consider $F(s, x+\lambda \xi)$ as a polynomial in one variable $\lambda$ with $s, x, \xi$ as parameters; let $\Delta_{i}^{F}(s, x, \xi)$ be its generalised discriminants. Let

$$
C_{i}=\left\{(s, \xi): \Delta_{i}^{F}(s, x, \xi)=0 \text { for all } x \in H\right\} .
$$

Let $j$ be the smallest number such that $\left(S \backslash S_{1}\right) \times H \nsubseteq C_{j}$; put $C=C_{j}, \Delta=\Delta_{j}^{F}$. If $\Delta(s, x, \xi)=\sum \Delta_{\alpha}(s, \xi) x^{\alpha}(x \in H)$, then $C$ is given by $\Delta_{\alpha}(s, \xi)=0$ for all $\alpha$. Let $\Delta_{\alpha}(s, \xi)=\sum \Delta_{\alpha \beta}(s) \xi^{\alpha}$; put $S_{0}=S_{1} \cup\left\{\Delta_{\alpha \beta}(s)=0\right.$ for all $\left.\alpha, \beta\right\}$. It is easy to see that $N Z\left(X_{s}\right)$ is given (for $\left.s \in S \backslash S_{0}\right)$ by $A_{\gamma}(s, x)=0$ for all $\gamma$, where

$$
\Delta(s, \xi, x)=\sum A_{\gamma}(s, x) \xi^{\gamma} .
$$


Now suppose that $d$ is arbitrary. Let $\Pi \subset H \times \ldots \times H(n-d-1$ times $)$ $=H^{n-d-1}$ be the set of all $a=\left(a_{d+2}, \ldots, a_{n}\right)$ such that $\partial / \partial x_{2}, \ldots, \partial / \partial x_{d+1}$, $a_{d+2}, \ldots, a_{n}$ are linearly independent. Every $a \in \Pi$ determines a projection $\Pi(a): C^{n} \rightarrow C^{d+1}$. There is a Zariski open set $\Omega \subset S \times \Pi$ and an algebraic set $\mathfrak{X} \subset S \times H^{n-d-1} \times C^{d+1}$ such that if $(s, a) \in \Omega$, then $\mathfrak{X}_{(s, a)}=\pi(a)\left(X_{s}\right)$ and $\operatorname{dim} \mathfrak{X}_{(s, a)}=d$. Choose a polynomial $P(s, x)$ such that the complement of $\Omega$ in $S \times H^{n-d-1}$ is contained in $\{P=0\}$. By the codimension 1 - case there exists an algebraic set $W \subset S \times H^{n-d-1}$, given by $Q_{i}(s, a)=0$, and an algebraic set $3 \subset S \times H^{n-d-1} \times C^{d+1}$ such that $\Omega \notin 3$ and $\mathcal{Z}_{(s, a)}=N Z\left(\mathfrak{X}_{(s, a)}\right)$ for $(s, a) \in \Omega \backslash W$. Let $S_{0}=\left\{s: P(s, a)=0, Q_{i}(s, a)=0\right.$ for all $i$ and for all $\left.a \in H^{n-d-1}\right\} \mp S$. Let $G_{i}(s, a, z)=0$ be the equations of 3 (where $z \in C^{+1}$ ). For $s \in S \backslash S_{0}$ we have: $x \in Z\left(X_{s}\right)$ if and only if $\pi(a) x \notin Z_{(s, a)}$ for an open set of $a^{\prime}$ s, so $N Z\left(X_{\mathrm{s}}\right)$ is given by $G_{j}(s, a, \pi(a) x)=0$ for all $a \in H^{n-d-1}$.

Corollary 3. Let $S \subset C^{p}, X \subset S \times C^{n}$ be algebraic such that all the fibers $X_{s}$ are equidimensional. Then

$$
N Z(X)=\bigcup_{s \in S}\left(\{s\} \times N Z\left(X_{s}\right)\right)
$$

is constructible.

Lemma 7. Let $S \subset C^{p}, X, Y \subset S \times C^{n}$ be algebraic. Then there exist

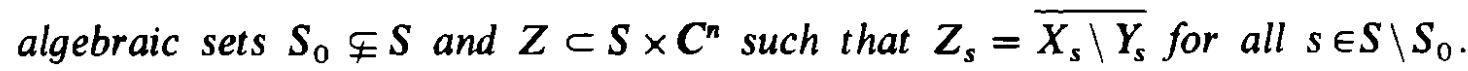

Proof. Take any non-zero $f \in I(X), g \in I(Y)$. After a linear change of coordinates in $C^{n}$ we can assume that $f=a(s) x_{n}^{k}+\ldots, g=b(s) x_{n}^{l}+\ldots$, where ... denote terms of lower degree with respect to $x_{n}$. Let $S_{1}$ $=\{s \in S: a(s)=0, b(s)=0\} \mp S$. Put $X_{s}^{\prime}=\pi_{0}\left(X_{s}\right)$, where $\pi_{0}: C^{n} \rightarrow C^{n-1}$ is the projection parallel to the $X_{n}$-axis. Note that $\pi_{0}: X_{s} \rightarrow X_{s}^{\prime}$ is proper for $s \notin S_{1}$ and therefore $X_{s}^{\prime}$ are algebraic for $s \notin S_{1}$. There exist an algebraic set $X^{*} \subset S \times C^{n-1}$ such that $X_{s}^{\prime}=X_{s}^{*}$ for $s \notin S_{1}$. There exists polynomials $\delta\left(s, x^{\prime}\right)$ and $\varphi(s, x)$ (where $\left.x^{\prime}=\left(x_{1}, \ldots, x_{n-1}\right)\right)$ such that: $1^{\circ} \delta$ does not vanish identically on $X^{*}, 2^{\circ}$ for every $\left(s, x^{\prime}\right) \in X^{*} \backslash\{\delta=0\}$ we have

$$
\left(s, x^{\prime}, x_{n}\right) \in X \backslash Y \Leftrightarrow \varphi\left(s, x^{\prime}, x_{n}\right)=0 .
$$

Put

$$
\tilde{X}_{s}=\left[X_{s} \cap \pi_{0}^{-1}\left(\left\{\delta_{s}=0\right\}\right)\right] \cup\left[X_{s} \cap\left\{\varphi_{s}=0\right\}\right],
$$

where $\delta_{s}\left(x^{\prime}\right)=\delta\left(s, x^{\prime}\right)$ and $\varphi_{s}(x)=\varphi(s, x)$. Clearly $\overline{X_{s} \backslash Y_{s}}=\overline{X_{s} \backslash Y_{s}}$ and $\tilde{X}=\bigcup_{s}\left[\{s\} \times \tilde{X}_{s}\right]$ is algebraic. If $\tilde{X}_{s} \mp X_{s}$ for some $s \notin S_{1}$, we can repeat the

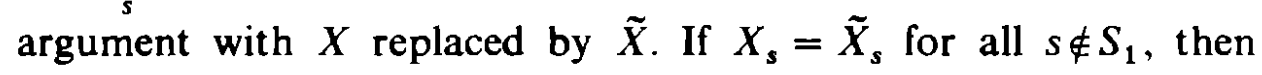

$$
\overline{X_{s} \backslash Y_{s}}=\overline{\left[X_{s} \cap \pi_{0}^{-1}\left(\left\{\delta_{s}=0\right\}\right)\right] \backslash Y_{s}} \cup\left[X_{s} \cap \pi_{0}^{-1}\left(\overline{X_{s}^{*} \backslash\left\{\delta_{s}=0\right\}}\right)\right]
$$

and the conclusion of the lemma can be assumed to hold for $\overline{\left[X_{s} \cap \pi_{0}^{-1}\left(\left\{\delta_{s}=0\right\}\right)\right] \backslash Y_{s}}$ and $\overline{X_{s}^{*} \backslash\left\{\delta_{s}=0\right\}}$. 
Corollary 4. $\bigcup_{s \in S}\left(\{s\} \times \overline{X_{s} \backslash Y_{s}}\right)$ is constructible.

By induction on $k$ we get now:

Corollary 5. Let $S \subset C^{p}, X, Y \subset S \times C^{n}$ be algebraic and all the fibres $X_{s}$ are equidimensional. Then, for all $i, a, k \bigcup_{s}\left[\{s\} \times\left(X_{s}\right)_{k}^{\wedge i a}\right], \bigcup_{s}\left[\{s\} \times\left(Y_{s}\right)_{k}^{\text {iia }}\right]$ are constructible ; in particular (putting $S=$ point) $X_{k}^{\wedge i a}, Y_{k}^{\vee i a}$ are constructible.

\section{Proofs of the corollaries}

Proof of Corollary 1. We observe first that $G_{K N}(X)$ is semialgebraic (if we consider $C^{n}$ and $G_{K N}$ as real vector spaces). In fact, the function

$$
X_{\text {reg }} \times X_{\text {reg }} \ni\left(q_{1}, q_{2}\right) \mapsto\left|P_{q_{1}}-P_{q_{2}}\right| \in R
$$

is semialgebraic (i.e. its graph is semialgebraic) and similarly the distance function $\left(q_{1}, q_{2}\right) \mapsto\left|q_{1}-q_{2}\right|$. We rewrite the definition of $G_{K N}(X)$ :

$$
\begin{gathered}
G_{K N}(X)=\left\{g: \exists \varepsilon>0, C>0 \forall q_{1}, q_{2} \in X_{\text {reg }},\left|q_{1}\right|<\varepsilon,\left|q_{2}\right|<\varepsilon, \exists x g_{1}(x)=0,\right. \\
\left.\ldots, g_{K}(x)=0,\left|P_{q_{1}}-P_{q_{2}}\right| \leqslant C\left|q_{1}-q_{2}\right| / \min \left(\left|q_{1}-x\right|,\left|q_{2}-x\right|\right)\right\} ;
\end{gathered}
$$

now our claim follows directly from Tarski's theorem (e.g. [2]).

Similarly the sets

$$
K_{k}^{i a}(X)=\left\{g: X_{k}^{\wedge i a} \subset\left(Y_{g}\right)_{k}^{\vee i a}\right\}
$$

are constructible since their definition can be rewritten as

$$
\left.g: \forall\left(p, p_{1}, \ldots, p_{k}\right)\left(p, p_{1}, \ldots, p_{k}\right) \in X_{k}^{\wedge i a} \Rightarrow\left(p, p_{1}, \ldots, p_{k}\right) \in\left(Y_{g}\right)_{k}^{\vee i a}\right\}
$$

and, because of Corollary 5, the results of [2] can be again used. So the semialgebraic set $G_{K N}(X)^{\prime}$ is a countable intersection $\bigcap_{i, a, k} K_{k}^{i a}(X)$ of constructible sets; this is possible, as is easy to see, only if the intersection stabilises. Thus, for sufficiently big $a, k$ e have

$$
G_{K N}(X)=\bigcap_{i, b \leqslant a, l \leqslant k} K_{l}^{i b}(X) .
$$

To prove that $G_{K N}^{\prime}(X)$ is constructible, we have only to observe that $\left\{g \in G_{K N}: \operatorname{dim} Y_{g} \leqslant r\right\}$ is constructible for every $r$; it is easily proved by induction on $n$.

Proof of Corollary 2. We prove as before that $L(X, Y)$ is semialgebraic. Now for every $p \in X_{\text {stng }}$ we put

$$
\begin{aligned}
& X_{k}^{\wedge i a}(p)=\left\{\left(p_{1}, \ldots, p_{k}\right):\left(p, p_{1}, \ldots, p_{k}\right) \in X_{k}^{\wedge i a}\right\}, \\
& Y_{k}^{\vee i a}(p)=\left\{\left(p_{1}, \ldots, p_{k}\right):\left(p, p_{1}, \ldots, p_{k}\right) \in Y_{k}^{\vee i a}\right\} .
\end{aligned}
$$


Again using [2] we prove that the sets

$$
L_{k}^{i a}=\left\{p \in X_{\text {sing }}: X_{k}^{\wedge i a} \subset Y_{k}^{\vee i a}\right\}
$$

are constructible. By our proposition $L(X, Y)$ is the interior in $X_{\text {sing }}$ of $\bigcap L_{k}^{i a} ;$ this implies, as is easy to see, that $L(X, Y)$ is constructible. It follows that $N L(X, Y)$ is constructible, and, since it is closed, it is algebraic.

\section{Examples}

We shall use our proposition to give some explicit examples of Lipschitz stratifications of surfaces in $C^{3}$.

Let $X$ be the germ at 0 given by

$$
y^{2}=x^{3}+z^{2} x^{2}
$$

We shall describe all curves $Y$ satisfying (*). Of course the only interesting point is the origin. Clearly

$$
C_{0}(X): y=0 \text {. }
$$

First we find tangents to $Y$ at 0 .

a) If we substitute $z x$ for $z$ and $z y$ for $y$, we get

$$
y^{2}=x^{2} z+x \text {. }
$$

The only $N Z$-point is $x=0, y=0, z=0$; it corresponds to the $z$-axis so the $z$-axis must be tangent to a component of $Y$.

b) If we substitute $x z$ for $z$ and $x y$ for $y$, we get

$$
y^{2}=x+x^{2} z^{2}
$$

and this surface has no $N Z$-points.

Thus we can assume that $Y$ is tangent to the $z$-axis. Now we take any integer $a \in N$ and put

$$
z=t^{a}, \quad x=t^{a+1} x_{1}, \quad y=t^{a+1} y_{1} .
$$

The strict transform of $X$ is

$$
X_{1}: y_{1}^{2}=t^{a+1} x_{1}^{2} t^{a-1}+x_{1}
$$

it has only one $N Z$-point: $x_{1}=0, y_{1}=0, t=0$. We have to substitute

$$
x_{1}=t x_{2}, \quad y_{1}=t y_{2} \text {. }
$$

The strict transform of $X_{1}$ is

$$
X_{2}: \quad y_{2}^{2}=t^{a+2} x_{2}^{2} t^{a-2}+x_{2} .
$$


The only $N Z$-point is again $x_{2}=0, y_{2}=0, t=0$, so we have to substitute

$$
x_{2}=t x_{3}, \quad y_{2}=t y_{3},
$$

etc. After $a$ such steps we get

$$
X_{a}: \quad y_{a}^{2}=t^{2 a} x_{a}^{2}\left(1+x_{a}\right) .
$$

$X_{a}$ has two $N Z$-points:

$$
\begin{gathered}
\text { I: } x_{a}=0, y_{a}=0, t=0, \\
\text { II: } \quad x_{a}=-1, y_{a}=0, t=0 .
\end{gathered}
$$

If we blow-up 1 , i.e. substitute

$$
x_{a}=t x_{a+1}, \quad y_{a}=t y_{a+1},
$$

we get

$$
X_{a+1}: \quad y_{a+1}^{2}=t^{2 a} x_{a+1}^{2}\left(1+t x_{a+1}\right)
$$

with the only $N Z$-point $x_{a+1}=0, y_{a+1}=0, t=0$, and the same situation will appear after any number of blowing-ups. So, remembering that for every $k$

$$
x=t^{a+k}, \quad y=t^{a+k} y_{k}, \quad z=t^{a},
$$

we see that $I$ corresponds to the $z$-axis, which must be a component $Y_{1}$ of $Y$. Now we consider II. We substitute

$$
x_{a}=-1+t x_{a+1}, \quad y_{a}=t y_{a+1}
$$

we get

$$
X_{a+1}: \quad y_{a+1}^{2}=t^{2 a-1}\left(-1+t x_{a+1}\right)^{2} x_{a+1} \text {; }
$$

the only $N Z$-point of $X_{a+1}$ is $x_{a+1}=0, y_{a+1}=0, t=0$. So we substitute

$$
x_{a+1}=t x_{a+2}, \quad y_{a+1}=t y_{a+2},
$$

etc. After $2 a$ such steps we get

$$
X_{3 a}: \quad y_{3 a}^{2}=\left(-1+t^{2 a} x_{3 a}\right)^{2} x_{3 a} ;
$$

this surface has no $N Z$-points, so the procedure stops. Thus $Y$ must contain a curve $Y_{2}$ on which

$$
x+t^{2 a} \equiv 0 \bmod t^{4 a}, \quad y \equiv 0 \bmod t^{4 a} .
$$

Such a curve can be of course characterised by

$$
Y_{2}: \quad x+z^{2}=\lambda z^{4}, y=\mu z^{4}, \quad \lambda, \mu \text { bounded }
$$

Thus finally any Lipschitz stratification of $X$ is

$$
X \supset(z \text {-axis }) \cup Y_{2} \supset\{0\} \text {. }
$$


As a second example we derive a relation between Lipschitz stratifications and polar curves. Let $X$ be a surface in $C^{3}$. Assume that the projection $\pi: C^{3} \rightarrow C^{2}$, parallel to the $z$-axis, is proper when restricted to $X$. For every $\xi \in C^{2}$ we have the projection $\pi(\xi): C^{3} \rightarrow C^{2}$, parallel to $(\xi, 1)$. Let $P(\xi)$ be the polar curve determined by $\pi(\xi)$, i.e.

$$
\begin{array}{r}
P(\xi)=\text { the closure of }\left\{x \in X_{\mathrm{reg}}: d \pi(\xi): T_{x} X \rightarrow T_{\pi(\xi) x} C^{2}\right. \\
\text { is not a linear isomorphisms }\} .
\end{array}
$$

There exists an open set $\Omega$ in $C^{2}$ such that the number of components of $P(\xi)$ for $\xi \in \Omega$ is independent of $\xi$ :

$$
P(\xi)=P_{1}(\xi) \cup \ldots \cup P_{\mu}(\xi),
$$

and the Puiseux expansion of every $P_{z}(\xi)$ has the form (after introducing $x_{1}$ $=x, x_{2}=y$ )

$$
\begin{aligned}
P_{a}(\xi): \quad x_{i} & =\varphi_{i}^{\alpha}\left(z^{1 / r}, \xi\right) \\
& =\sum_{j=1}^{j(i, x)-1} a_{i j}^{\alpha} z^{j / r}+b_{i}^{\alpha}(\xi) z^{j(i, a) / r}+o\left(z^{j(i, a) / r}\right)
\end{aligned}
$$

where $\varphi_{i}^{\alpha}$ are analytic in $z, \xi, a_{i j}=$ const (independent of $\xi$ ) and $b_{i}^{\alpha}(\xi) \neq$ const at least for one $i$ (remark that $j(i, \alpha)$ is finite at least for one $i$, for every $\alpha$ ).

For every $\alpha$ let $j(\alpha)=\min _{i=1,2} j(i, \alpha)$ and

$$
Y_{\alpha}: \quad x_{i}=\sum_{j=1}^{j(\alpha)} a_{i j}^{\alpha} z^{j / r}+o\left(z^{j(\alpha) / r}\right), \quad i=1,2,
$$

where, of course, $o\left(z^{j(\alpha) / r}\right)$ denotes any function going faster to 0 than $z^{j(a) / r}$.

We shall prove that for any choice of the "remainders" $o\left(z^{j(x) / r}\right)$ the curve $Y$ defined by

satisfies $(*)$.

$$
Y=X_{\text {sing }} \cup Y_{1} \cup \ldots \cup Y_{\mu}
$$

It is enough to show that for any two curves $q_{1}(t), q_{2}(t)$, lying in $X_{\text {reg }} \backslash Y$ for $t \neq 0$, such that

$$
\operatorname{ord}\left|q_{1}(t)-q_{2}(t)\right|>\text { ord } d\left(q_{1}(t), Y\right)
$$

(*) holds, with $C$ depending maybe on these curves.

So let $q_{1}(t), q_{2}(t)$ be such curves. Then we remark that there exists a number $c>0$ and an open and non-empty set $\Omega_{0} \subset \Omega$ such that for all $\xi \in \Omega_{0}$

$$
d\left(q_{1}(t), X_{\text {sing }} \cup P(\xi)\right) \geqslant c d\left(q_{1}(t), Y\right) \text { for all } t \text { sufficiently close to } 0 .
$$

We change coordinates. Let $\bar{x}_{1}$ be any axis such that, for some $c^{\prime}>0$,

$$
\Varangle\left(\partial / \partial \bar{x}_{1}, \dot{q}_{1}(t) /\left|\dot{q}_{1}(t)\right|\right)<\frac{1}{2} \pi-c^{\prime}
$$


Take any $\xi_{0} \in \Omega_{0}$ such that $\partial / \partial \bar{x}_{1} \notin \operatorname{ker} \pi\left(\xi_{0}\right)$ and let ker $\pi\left(\xi_{0}\right)$ be the direction of the $\bar{x}_{3}$-axis. The $\bar{x}_{2}$-axis we choose arbitrarily. We can suppose that $q_{1}(0)$ $=q_{2}(0)=0$.

We take for $H$ the plane $\bar{x}_{1}=0$ and define $T_{q}^{0} X, P_{q}^{0}$ etc. as before. Thus we have to prove that

$$
\left|P_{q_{1}(t)}^{0}-P_{q_{2}(t)}^{0}\right| \leqslant C\left|q_{1}(t)-q_{2}(t)\right| / d\left(q_{1}(t), Y\right) .
$$

Let us take an integer $N$ such that if

$$
\psi\left(\bar{x}_{1}, \bar{x}_{2}, \bar{x}_{3}\right)=\left(\bar{x}_{1}^{N}, \bar{x}_{2}, \bar{x}_{3}\right),
$$

then $\psi^{-1}\left(q_{i}(t)\right)$ have branches which can be described by

$$
\tilde{q}_{i}=\tilde{q}_{i}\left(\bar{x}_{1}\right): \quad \bar{x}_{j}=g_{j}^{(i)}\left(\bar{x}_{1}\right), \quad j=2,3 ; i=1,2,
$$

$g_{j}^{(i)}$ analytic, and further

$$
\text { ord } d\left(\tilde{q}_{1}\left(\bar{x}_{1}\right), \psi^{-1}(Y)\right)=b \in N .
$$

Put

$$
\bar{x}_{j}=g_{j}^{(1)}\left(\bar{x}_{1}\right)+u_{j} \bar{x}_{1}^{b}, \quad j=2,3,
$$

where $u_{2}, u_{3}$ are new variables. Thus we have maps

$$
C_{\left(\bar{x}_{1}, u_{2}, u_{3}\right)}^{3} \stackrel{\varphi}{\rightarrow} C^{3} \stackrel{\Psi}{\rightarrow} C^{3} .
$$

Let $H^{\prime} \subset C_{\left(\bar{x}_{1}, u_{2}, u_{3}\right)}^{3}$ be given by $\bar{x}_{1}=0$ and

$$
X^{*}=\overline{(\varphi \psi)^{-1}(X) \backslash H^{\prime}} ;
$$

let

$$
q_{i}^{*}: \quad u_{j}=h_{j}^{(i)}\left(\bar{x}_{1}\right), \quad j=2,3 ; i=1,2,
$$

be the equations of the curves $\tilde{q}_{i}$ in the $\left(\bar{x}_{1}, u_{2}, u_{3}\right)$-coordinates.

Now if we take a projection $\pi(\xi)$, where $\xi \in \Omega_{0}$ and ker $\pi(\xi)$ contains a vector $\alpha\left(\partial / \partial \bar{x}_{2}\right)+\beta\left(\partial / \partial \bar{x}_{3}\right), \alpha \neq 0$ or $\beta \neq 0$, then

$P^{*}(\xi) \stackrel{\text { der }}{=}$ the polar variety of $X^{*}$ defined by the linear projection whose kernel contains $\alpha\left(\partial / \partial u_{2}\right)+\beta\left(\partial / \partial u_{3}\right)$ $=(\varphi \psi)^{-1}(P(\xi)) \subset H^{\prime}$,

by the choice of $b$. The set of projections in the $C_{\left(\bar{x}_{1}, u_{2}, u_{3}\right)}^{3}-$ space for which the above formula holds, is of course open in the set of all projections parallel to $H^{\prime}$ (with one-dimensional kernel). This implies that $X^{*}$ has no $N Z$-points. Thus

$$
\left|P_{q_{1}^{*}\left(\bar{x}_{1}\right)}^{0}-P_{q_{2}^{*}\left(\bar{x}_{1}\right)}^{0}\right| \leqslant C\left|q_{1}^{*}\left(\bar{x}_{1}\right)-q_{2}^{*}\left(\bar{x}_{1}\right)\right|,
$$

so

$$
\left|P_{q_{1}(t)}^{0}-P_{q_{2}(t)}^{0}\right| \leqslant C\left|q_{1}(t)-q_{2}(t)\right| /|t|^{b}
$$




\section{References}

[1] T. Mostowski, Lipschitz equisingularity, Dissertationes Math. 243 (1985).

[2] A. Robinson, Introduction to Model Theorv, North-Holland. Amsterdam 1963.

[3] B. Teissier, Variétés polaires II, in Actes de la Conférence de géometrie algébrique à la Ràbida, Lecture Notes in Math. 961, Springer-Verlag, Berlin 1981, 314-491.

[4] H. Whitney, Complex Analytic Varieties, Addison-Wesley, Reading 1972.

Presented to the semester

Singularities

15 February-15 June, 1985 